\title{
Visual prepotency and eye orientation
}

\author{
FRANCIS B. COLAVITA, ROSEMARY TOMKO, and DANIEL WEISBERG \\ University of Pittsburgh, Pittsburgh, Pennsylvania 15260
}

\begin{abstract}
When simultaneously presented with a brief auditory and a brief visual stimulus of equal subjective magnitude, human subjects show a strong tendency to respond to the visual stimulus. The present experiments attempted to reduce or abolish this prepotency effect by manipulating the subject's visual fixation point, based upon the idea that eye orientation plays an important part in attending to either a brief auditory or a brief visual stimulus. The results suggested that visual prepotency in human subjects persists even when peripheral rather than foveal vision is used.
\end{abstract}

A recent series of experiments (Colavita, 1974) has demonstrated a strong visual prepotency in human subjects when they are placed in a conflict situation where they must respond to either a brief light stimulus or a brief tone stimulus. In these experiments, subjects matched an auditory and a visual stimulus for subjective magnitude. Then, each stimulus was used as a cue in a reaction time (RT) task. On occasions when both stimuli were presented simultaneously, the subject's responding was seen to be dominated by the visual stimulus even when verbal instructions were given to respond to the tone in such instances. An interesting secondary finding was the fact that on some of the trials involving simultaneous lighttone presentations subjects were unaware that the tone had been presented.

An attempt was made to explain the above results by referring to Broadbent's (1958) channel-switching model of information processing and attention. This model predicts that two simultaneously presented stimuli cannot be processed as effectively as the same two stimuli presented in succession. However, additional explanation is required for the fact that the visual channel is apparently sampled first by human subjects.

We have proposed that for simultaneously presented auditory and visual stimuli of short duration, the resulting reflexive orienting response would more likely favor the visual rather than the auditory input. This proposal was based upon some evidence (Ades, 1944) that in the case of brief auditory and visual stimuli, the orienting response may ultimately involve the neural connections of the superior colliculus with the motor centers of the ventral tegmentum, medulla, and spinal cord. Thus, the visual channel might be sampled first by virtue of its more direct connections with the superior colliculus.

Implicit in the above proposal is the idea that eye

This research was supported by USPHS Grant No. NS-09027 from the National Institute of Neurological and Communicative Diseases and Stroke. orientation plays an integral part in attending to either a brief auditory stimulus or a brief visual stimulus. Thus, the visual prepotency noted in our RT task may be due to the fact that subjects are already fixating their gaze on the light source in anticipation of its being activated. The two experiments reported below attempt to manipulate visual prepotency by altering subjects' visual fixation point.

\section{EXPERIMENT I}

Experiment I was designed to measure sensory prepotency in a choice RT situation when the subject was instructed to use as a visual fixation point a spot midway between the sound source and the light source.

\section{Method}

Ten freshman psychology students at the University of Pittsburgh served as subjects. The apparatus and procedure have been described elsewhere (Colavita, 1974). Briefly, each subject matched an auditory and a visual stimulus for subjective intensity by adjusting a 6 -W light source until it was as bright as a $4,000-\mathrm{Hz}$ 65-dB SPL tone was loud. Then, the matched stimuli served as cues in a RT task. Each subject was seated in front of a stimulus panel containing a $5-\mathrm{cm}$ speaker and the $6-\mathrm{W}$ incandescent light bulb. In contrast to our earlier work, subjects were instructed to look at a fixation point midway between the light and the speaker, which were $25 \mathrm{~cm}$ apart. Then, each subject was told to press one of two telegraph keys when the tone was presented, and the other key when the light was activated. Subjects were informed that on some trials both light and tone would be presented simultaneously. On these trials, the subject was told to press whichever key was appropriate to the signal recognized first.

Subjects were given 30 simple RT trials when they were told ahead of time which stimulus would occur. Then 30 choice RT trials were presented when the subject was not told ahead of time which stimulus would be activated. Interspersed with the 30 choice RT trials were five conflict trials when the tone and the light were presented simultaneously. There were 50 such conflict trials altogether (10 subjects, five trials each).

\section{Results}

Mean simple RTs were $269 \mathrm{msec}$ and $271 \mathrm{msec}$ for tone and light trials, respectively. Mean choice RTs were $331 \mathrm{msec}$ and $326 \mathrm{msec}$. Out of 50 conflict trials, the light key was pressed on 42 occasions. On two of 
these 42 occasions, subjects were unaware that the tone had in fact been presented.

\section{EXPERIMENT II}

In a further attempt to manipulate the apparent prepotency of the visual stimulus, subjects' visual fixation point was moved even farther from the light source, with subjects now being instructed to fixate on the speaker for the duration of the experiment.

\section{Method}

Ten freshman psychology students from the University of Pittsburgh served as subjects. The apparatus and procedure remained the same as in Experiment $I$, with the exception that the subject was now told to use the speaker as a visual fixation point.

\section{Results}

Mean simple RTs were 231 and $226 \mathrm{msec}$ for tone and light, respectively. These means were not significantly different. Mean RTs in the choice situation were $319 \mathrm{msec}$ for tone trials and $318 \mathrm{msec}$ for light trials. Out of the 50 conflict trials, light keypresses occurred 38 times with tone keypresses occurring on 12 trials. One subject was unaware on two conflict trials that the tone had been presented.

\section{DISCUSSION}

The results of the above two experiments are in agreement with our earlier finding of a strong visual prepotency in human subjects when brief auditory and visual stimuli are presented simultaneously (Colavita, 1974). Our experimental hypothesis in conducting the above two experiments was that we could reduce visual prepotency by manipulating subjects' visual fixation point. This hypothesis grew out of the idea that the orienting response to either a brief auditory stimulus or a brief visual stimulus is mediated by the neural connections of the superior colliculus with the motor centers of the ventral tegmentum, medulla, and spinal cord. Our expectations were only partially realized. In Experiment I, with subjects fixating midway between the light source and the speaker, eight tone keypresses were noted out of 50 conflict trials; in Experiment II, 12 tone keypresses occurred with the subject fixating on the speaker. When these ratios are compared with the three tone keypresses out of 50 trials (reported in our earlier work) with subjects fixating on the light source, a trend appears suggesting that eye fixation plays a small but measureable part in the prepotency of the visual stimulus. However, the visual stimulus clearly continues to dominate subject's responding, even when the sound source is used as a visual fixation point. Light keypresses were noted on 38 out of 50 trials under this condition.

Two instances each in Experiment I and Experiment II of unawareness of the presence of the tone on conflict trials are further evidence of the prepotency of the visual stimulus. It would appear that even when peripheral rather than foveal vision is used, human subjects show a strong visual prepotency when brief auditory and visual stimuli occur simultaneously.

\section{REFERENCES}

Ades, H. W. Midbrain auditory mechanisms in cats. Journal of Neurophysiology, 1944, 7, 415-424.

Brondbent, D. E. Perception and communication. London: Pergamon Press, 1958.

Colavita, F. B. Human sensory dominance. Perception \& Psychophysics, 1974, 16, 409-412.

(Received for publication December 17, 1975.) 